\title{
A Novel Triangular Element with Continuous Nodal Acoustic Pressure Gradient for Acoustic Scattering Problems
}

\author{
Yingbin Chai $\mathbb{D}^{1,2,3}$ Wei Li $\mathbb{D}^{1}{ }^{1}$ and Yong-Ou Zhang $\mathbb{D}^{1,4}$ \\ ${ }^{1}$ School of Naval Architecture and Ocean Engineering, Huazhong University of Science and Technology, Wuhan, \\ Hubei 430074, China \\ ${ }^{2}$ Collaborative Innovation Center for Advanced Ship and Deep-Sea Exploration (CISSE), Shanghai 200240, China \\ ${ }^{3}$ School of Mechanical Science \& Engineering, Huazhong University of Science and Technology, Wuhan, Hubei 430074, China \\ ${ }^{4}$ School of Transportation, Wuhan University of Technology, Wuhan 430063, China \\ Correspondence should be addressed to Yong-Ou Zhang; zhangyo@whut.edu.cn
}

Received 9 July 2019; Accepted 19 September 2019; Published 16 October 2019

Academic Editor: Fausto Arpino

Copyright (C) 2019 Yingbin Chai et al. This is an open access article distributed under the Creative Commons Attribution License, which permits unrestricted use, distribution, and reproduction in any medium, provided the original work is properly cited.

To improve the performance of the standard finite element (FE) method in acoustic simulation, a novel triangular element with continuous nodal acoustic pressure gradient (FEM-T3-CNG) is presented to solve two-dimensional underwater acoustic scattering problems. In this FEM-T3-CNG model, the local approximation (LA) is represented by using the least-squares (LS) scheme, and the standard FE shape functions are employed to satisfy the partition of unity (PU) concept. In order to possess the important delta Kronecker property, the constrained orthonormalized LS (CO-LS) is utilized to construct the hybrid nodal shape functions. Incorporating the present FEM-T3-CNG element with the proper nonreflecting boundary condition, the two-dimensional underwater acoustic scattering problems in the infinite domain could be solved ultimately. The numerical results show that the present FEM-T3-CNG element behaves much better than the standard FEM-T3 element in terms of computation accuracy and can be regarded as a good alternative approach in exterior acoustic computation.

\section{Introduction}

The acoustic scattering by underwater objects is a very important physical phenomenon in acoustics and its main concern is how the acoustic wave is affected by the objects standing in its propagation path. The underwater acoustic scattering has a very strong background in many practical engineering application fields, such as ocean resource exploration, sound navigation and ranging, and underwater collision prevention and rescue to name a few. Therefore, how to precisely calculate and predict the acoustic scattered field is of great importance to improve the acoustical properties of the ocean engineering structures.

The essence of solving the acoustic scattering numerically is to handle the typical exterior boundary-value problems governed by the Helmholtz equation. Much research efforts have been devoted to such problems during the past few decades and they are still ongoing today. Actually, for the objects with relatively simple geometry shapes (such as cylinder and sphere), the exact solutions to acoustic scattering can be reached by using several analytical or semianalytical approaches. However, when it comes to the objects with very complicated geometry shapes, we have to resort to the pure numerical techniques. Among numerous numerical methods for exterior acoustic problems, the finite element method (FEM) [1] and the boundary element method (BEM) [2,3] have become two most preferable tools to deal with the exterior acoustic problems.

The BEM is essentially a boundary discretization technique, and the system matrices obtained from the BEM are asymmetric and nonsparse due to the nonlocal feature of the $\mathrm{BEM}$, giving rise to the rapidly increasing computational cost with the increment of nodes on the boundary. In addition, the classical BEM may produce nonunique solutions for exterior acoustic problems in certain wave numbers, and it is also difficult to deal with the anisotropic and nonhomogeneous acoustic media. Recently, an innovative and meshless singular boundary method (SBM) [4-7], which is 
quite different with the classical boundary integral method [8], has been devised to tackle acoustic problems. In SBM, the origin intensity factor is introduced to modify the fundamental solution $[9,10]$. Actually, the SBM is a typical boundary-based meshless numerical technique [11-14] and can effectively overcome some defects of the classical BEM. Currently, the SBM has been successfully applied in many engineering problems, such as the seismic wave problems [15] and electromagnetic wave problems [16].

Another alternative numerical approach for acoustic scattering computation is the classical finite element method (FEM). In contrast to the BEM, one attractive advantage of the FEM for exterior acoustic problems is that the obtained system matrices are usually sparse and symmetric though the number of the associated algebraic equations for the FEM is always larger than that for the BEM. These good features are beneficial to the decrease of the required storage memory and then to improve the computational efficiency. Actually, it is found that the FEM usually surpasses the BEM in terms of computational efficiency for the relatively simple acoustic scattering problems [17]. However, it is quite important to point out that the pure FEM cannot be employed directly to solve the exterior acoustic problems because it is a typical domain-based numerical approach, while the involved problem domain for the considered exterior acoustic problems is always infinite and unbounded. Nevertheless, a lot of numerical treatments, such as the Dirichlet-to-Neumann (DtN) [18-21] boundary condition, can be used to truncate the unbounded problem domains and enable the classical FEM to handle the exterior problems successfully.

In practice, the main stumbling block of the classical FEM for acoustic scattering computation is the numerical dispersion issue [22-27]. The accuracy of the FEM solutions for acoustic scattering is severely affected by the numerical dispersion. More importantly, the numerical error resulting from the numerical dispersion will accumulate with the increasing frequency values. Consequently, the classical FEM can only reach considerably accurate solutions in the relatively low-frequency range, and for the relatively highfrequency range, the corresponding solutions from the FEM are always not reliable.

Much research efforts have been performed to suppress the dispersion error and improve the quality of FEM solutions for acoustic computation, and many advanced FEM or meshless techniques [28-31] have been developed, such as Galerkin least-squares FEM [32], the smoothed FEM [3337], the mass-redistributed FEM [38], the overlapping finite elements [39], and so on. Though the improvements addressing the dispersion error have been reached to a certain extent by these methods, they still suffer from their own limits, either in unsatisfactory performance in controlling the dispersion error or the relatively complicated formulation. Practically, the numerical dispersion is still an unsolved issue for the classical FEM, and the related research work is still ongoing.

The objective of this work is to present a coupled "FEMeshfree" method for underwater acoustic scattering problems. In this novel coupled method, the FE shape functions from the standard linear triangular (T3) is combined with the least-squares (LS) scheme to form a composite shape function for the new element [40]. Since the derivatives of shape functions for this element are continuous at the nodes, the obtained acoustic pressure gradient field is also continuous at nodes, and then this element is named as the FEM-T3-CNG element in this work. In this FEM-T3-CNG element, the FE shape functions are used to satisfy the partition of unity (PU) concept, and the local approximation (LA) is represented by using the leastsquares (LS) scheme. As a consequence, both individual strengths of the two methods are captured and synergized by the present coupled method. By incorporating with the approximate nonreflecting boundary condition, the present coupled "FE-Meshfree" FEM-T3-CNG element can be ultimately employed to tackle the underwater scattering problems. The numerical results show that the present method significantly surpasses the standard FEM for acoustic scattering computation in terms of computation accuracy and computation efficiency. Therefore, the present approach exhibits great potentials in practical underwater acoustic scattering prediction.

\section{Basic Formulation for the Exterior Acoustic Problems}

In an ideal homogeneous isotropic medium, the acoustic wave equation is given by

$$
\nabla^{2} p+k^{2} p=f
$$

in which $\nabla^{2}$ represents the Laplace operator, $p$ is the acoustic pressure, and $f$ and $k$ stand for the source term and wave number, respectively.

For the exterior acoustic problems in the infinite problem domain, there are no spurious reflecting waves from the far field and the following Sommerfeld radiation condition should be obeyed [18]:

$$
\lim _{r \longrightarrow \infty} r^{(d-1) / 2}\left(\frac{\partial p}{\partial r}-i k p\right)=0,
$$

in which $d$ represents the space dimensions.

In order to tackle the exterior acoustic problems using the domain-based numerical techniques (such as the FEM), the infinite problem domain should be always truncated by an artificial boundary $B$ and a finite computational domain is then obtained (see Figure 1). In this work, the Dirichlet-toNeumann (DtN) boundary condition [18] is used on the artificial boundary $B$, namely,

$$
\frac{\partial p}{\partial n}=-M p \quad \text { on } B
$$

in which $M$ stands for the DtN operator.

Using the standard weighted residual techniques, the exterior acoustic wave equation can be formulated in the following matrix form [18]:

$$
\left\{\mathbf{K}-k^{2} \mathbf{M}+\mathbf{K}_{A B}^{b}\right\}\{\mathbf{P}\}=\{\mathbf{F}\},
$$




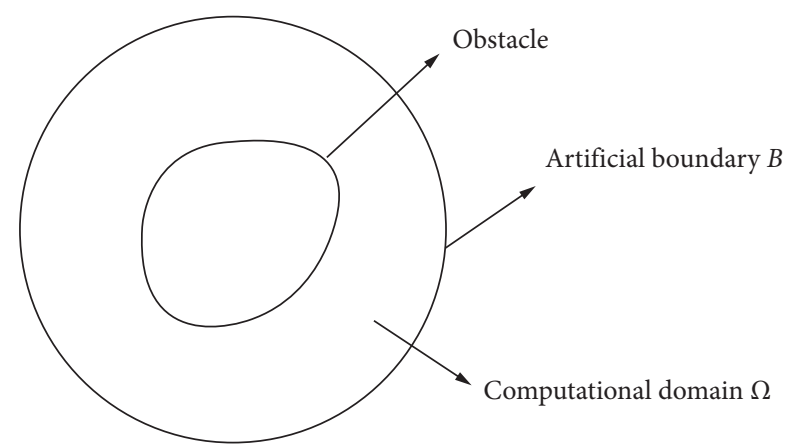

FIGURE 1: The infinite domain is usually truncated by an artificial boundary $B$ yielding a finite computational domain $\Omega$.

in which $\mathbf{K}$ and $\mathbf{M}$ are the corresponding stiffness matrix and mass matrix for acoustic problems; $\mathbf{P}$ and $\mathbf{F}$ represent the unknown nodal acoustic pressure and nodal force vector; and $\mathbf{K}_{A B}^{b}$ is a matrix corresponding to the $\mathrm{DtN}$ operator.

\section{Formulation of the FEM-T3-CNG Element}

For the local approximation in this work, the well-known least squares (LS) is employed. In the LS formulation, an unknown field function $u(\mathbf{x})$ in the considered problem domain is expressed by [40]

$$
u_{i}(\mathbf{x})=\mathbf{p}^{T}(\mathbf{x}) \mathbf{A}^{-1} \mathbf{B a},
$$

in which $\mathbf{p}(\mathbf{x})$ is the used local basis functions which are usually constructed using the monomials from the Pascal triangle; a represents a vector of nodal displacements (acoustic pressure in this paper) defined by

$$
\mathbf{a}^{T}=\left[\begin{array}{llll}
a_{1} & a_{2} & \cdots & a_{n^{[i]}}
\end{array}\right]^{T},
$$

in which $n^{[i]}$ denotes the number of nodes in the node support $\Omega_{i}$.

Following the classical LS formulation, we have

$$
\begin{aligned}
\mathbf{A} & =\sum_{j=1}^{n^{[i]}} \mathbf{p}\left(\mathbf{x}_{j}\right) \mathbf{p}^{T}\left(\mathbf{x}_{j}\right), \\
\mathbf{B} & =\left[\begin{array}{llll}
\mathbf{p}\left(\mathbf{x}_{1}\right) & \mathbf{p}\left(\mathbf{x}_{2}\right) & \cdots & \mathbf{p}\left(\mathbf{x}_{n^{[i]}}\right)
\end{array}\right] .
\end{aligned}
$$

To enhance the abilities of the aforementioned formulation, the constrained and orthonormalized least squares (CO-LS), in which the Gram-Schmidt orthonormalization process is included, is employed here. In the CO-LS, the orthonormalized vector $\mathbf{p}$ has the following form [40]:

$$
\mathbf{s}=\left[\begin{array}{llll}
s_{1} & s_{2} & \cdots & s_{m}
\end{array}\right]^{T}=\mathbf{R} \mathbf{p},
$$

in which the dimension of the orthogonalizing matrix $\mathbf{R}$ is $m \times m$ and $m$ stands for the number of the used monomials in $\mathbf{p}(\mathbf{x})$.

By normalizing the vector s, we have

$$
\mathbf{r}=\mathbf{H p},
$$

in which the orthonormalizing matrix $\mathbf{H}$ also has the dimension $m \times m$.
The constrained nodal approximation $u_{i}(\mathbf{x})$ can be then expressed by the following form by using the Lagrange multiplier method [40]:

$$
\begin{aligned}
& u_{i}(\mathbf{x})=\sum_{j=1}^{n^{[i]}} \widehat{\phi}_{j}^{[i]}(\mathbf{x}) a_{j} \\
& \widehat{\Phi}=\left[\begin{array}{lllll}
\widehat{\phi}_{1}^{[i]}(\mathbf{x}) & \widehat{\phi}_{2}^{[i]}(\mathbf{x}) & \cdots & \widehat{\phi}_{n^{[i]}}^{[i]}(\mathbf{x})
\end{array}\right]^{T}, \\
& \mathbf{B}^{[i]}=\left[\begin{array}{llll}
\mathbf{B}_{1}^{[i]} & \mathbf{B}_{2}^{[i]} & \cdots & \mathbf{B}_{n^{[i]}}^{[i]}
\end{array}\right], \\
& \mathbf{B}_{k}^{[i]}=\mathbf{r}\left(\mathbf{x}_{k}\right)-f_{k}^{[i]} \mathbf{r}\left(\mathbf{x}_{i}\right) \quad\left(k=1,2, \ldots, n^{[i]}\right), \\
& f_{k}^{[i]}=\left\{\begin{array}{l}
\frac{\sum_{j=1}^{m}\left(r_{j i} r_{j k}\right)}{\sum_{j=1}^{m} R_{j i}^{2}(k \neq i)}, \\
\frac{\left[\sum_{j=1}^{m}\left(r_{j i} r_{j k}\right)-1\right]}{\sum_{j=1}^{m} r_{j i}^{2}(k=i)},
\end{array}\right.
\end{aligned}
$$

in which $\widehat{\phi}_{j}^{[i]}(\mathbf{x})$ denotes the nodal shape function for node $j$.

In the present FEM-T3-CNG element, the approximation $u_{h}(\mathbf{x})$ is written by

$$
u_{h}(\mathbf{x})=w_{1}(\mathbf{x}) u_{1}(\mathbf{x})+w_{2}(\mathbf{x}) u_{2}(\mathbf{x})+w_{3}(\mathbf{x}) u_{3}(\mathbf{x}),
$$

in which $u_{i}(\mathbf{x})(i=1,2,3)$ are the local nodal approximation functions and $w_{i}(\mathbf{x})(i=1,2,3)$ are the weight functions.

$$
\begin{aligned}
& w_{1}(\mathbf{x})=N_{1}+N_{1}^{2} N_{2}+N_{1}^{2} N_{3}-N_{1} N_{2}^{2}-N_{1} N_{3}^{2}, \\
& w_{2}(\mathbf{x})=N_{2}+N_{2}^{2} N_{3}+N_{2}^{2} N_{1}-N_{2} N_{3}^{2}-N_{2} N_{1}^{2}, \\
& w_{3}(\mathbf{x})=N_{3}+N_{3}^{2} N_{1}+N_{3}^{2} N_{2}-N_{3} N_{1}^{2}-N_{3} N_{2}^{2},
\end{aligned}
$$

in which $N_{i}(i=1,2,3)$ are the standard linear nodal shape functions in the linear FEM-T3 element.

By substituting equations (12)-(14) into equation (11), the global approximation in the present FEM-T3-CNG element could be further expressed by

$$
u_{h}(\mathbf{x})=\sum_{i=1}^{3} w_{i}(\mathbf{x}) \sum_{j=1}^{n^{[i]}} \hat{\phi}_{j}^{[i]}(\mathbf{x}) a_{j} .
$$

Following the aforementioned formulation, the reconstructed nodal shape functions can be obtained easily and straightforward, and then the considered problem can be solved as similarly as in the standard FEM. However, it should be pointed that the derivatives of the nodal shape functions are continuous at nodes [40]; therefore, the obtained acoustic field using the present FEM-T3-CNG element possesses the continuous nodal acoustic pressure gradient. The numerical examples in the next section will be conducted to examine the abilities of the present FEM-T3CNG element for exterior acoustic computation.

\section{Numerical Results}

4.1. Dispersion Error Analysis. For solving the acoustic problems using numerical methods, one important issue we 
have to confront is the numerical dispersion error induced by the numerical discretization. The quality of the numerical solutions is severely affected by this dispersion issue. Generally, the used numerical method is able to provide relatively accurate solutions if it could effectively suppress the numerical discretization error. In this subsection, the dispersion error analysis will be performed to examine the abilities of the proposed methods in solving underwater acoustic scattering problems.

Following the previous formulation, the matrix form of the governing equation for acoustic problems is given by

$$
\overline{\mathbf{M}} \ddot{\mathbf{p}}+c^{2} \overline{\mathbf{K}} \mathbf{p}=\mathbf{0},
$$

in which the overdot stands for the time derivative and $\overline{\mathbf{M}}$ and $\overline{\mathbf{K}}$ represent the dimensionless mass matrix and stiffness matrix, respectively [39].

Using the Bathe time integration technique [39] for temporal discretization, we have

$$
\begin{aligned}
\left(72 \overline{\mathbf{M}}+8 c^{2} \Delta t^{2} \overline{\mathbf{K}}\right) \mathbf{p}^{t+\Delta t}+\left(-144 \overline{\mathbf{M}}+5 c^{2} \Delta t^{2} \overline{\mathbf{K}}\right) \mathbf{p}^{t+(\Delta t / 2)} \\
+\left(72 \overline{\mathbf{M}}+5 c^{2} \Delta t^{2} \overline{\mathbf{K}}\right) \mathbf{p}^{t}=\mathbf{0}
\end{aligned}
$$

in which $\Delta t$ is the time step.

Assuming that the numerical solutions of the considered acoustic problems have the following form:

$$
\mathbf{p}=A e^{j\left(k_{h} \mathbf{n} \cdot \mathbf{x}_{h}-\omega_{h} t_{h}\right)},
$$

in which $A$ is the acoustic pressure amplitude, $k_{h}$ is the numerical wave number, $\mathbf{x}_{h}$ is the discretized position vector, $\omega_{h}$ is the numerical angular frequency, and $t_{h}$ stands for the discretized time.

Substituting equation (18) into equation (17), we have

$$
\begin{gathered}
\left(\mathbf{D}_{\text {stiff }}-\lambda \mathbf{D}_{\text {mass }}\right) A=0, \\
\lambda=\frac{144\left[1-\cos \left(\omega_{h} \Delta t / 2\right)\right]}{C F L^{2} h^{2}\left[5+13 \cos \left(\omega_{h} \Delta t / 2\right)-j 3 \sin \left(\omega_{h} \Delta t / 2\right)\right]},
\end{gathered}
$$

in which $\mathbf{D}_{\text {stiff }}$ and $\mathbf{D}_{\text {mass }}$ are the resultant Hermitian matrices corresponding to $\overline{\mathbf{K}}$ and $\overline{\mathbf{M}}$, respectively. CFL is a parameter defined by $\mathrm{CFL}=c \Delta t / h$.

The acoustic pressure amplitude decay is related to the complex angular frequency $\omega_{h}$, namely,

$$
\zeta=\left(1-e^{\operatorname{Im}\left(\omega_{h} \Delta t\right)}\right) \times 100 \%
$$

in which $\zeta$ is the acoustic pressure amplitude decay per $\Delta t$.

If the nontrivial solutions to equation (19) exist, we have

$$
\operatorname{det}\left(\mathbf{D}_{\text {stiff }}-\lambda \mathbf{D}_{\text {mass }}\right)=0 \text {. }
$$

Using the aforementioned equation, the relationship between the numerical acoustic wave speed $c_{h}\left(\omega_{h} / k_{h}\right)$ and the exact one $c$ can be obtained.

In this work, the dispersion error analysis is performed based on the uniform mesh pattern, as shown in Figure 2.

The dispersion error, which is denoted by the ratio of numerical acoustic wave speed to exact acoustic wave speed

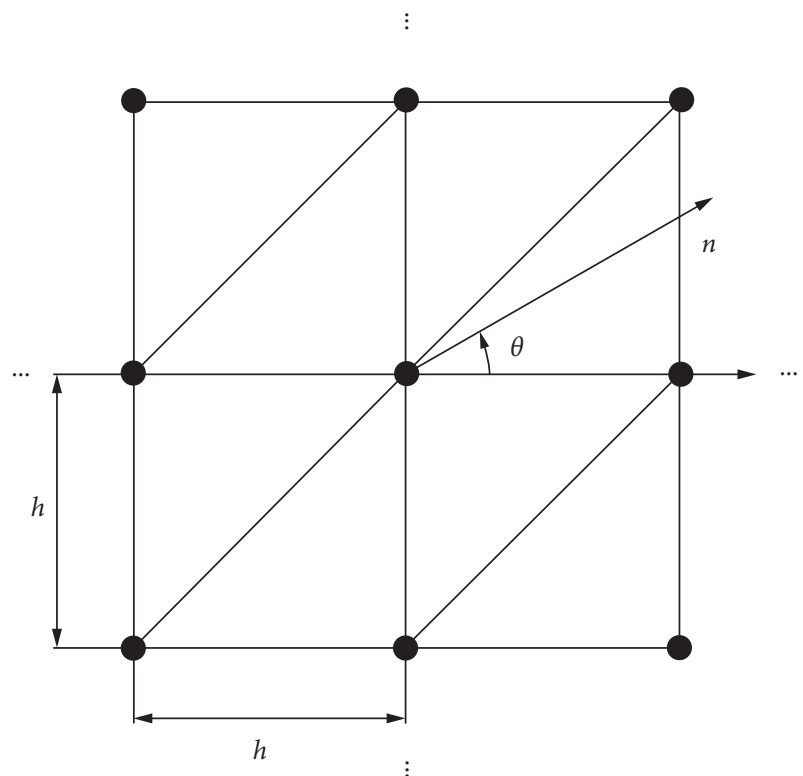

Figure 2: The uniform mesh pattern used for dispersion error analysis.

$c_{h} / c$, is shown in Figure 3. For comparison, the numerical results from the present FEM-T3-CNG element together with those from the standard FEM-T3 element are presented in the figures. It can be found from the figures that the dispersion errors from the two methods are different in the different propagation directions. However, the error results from the present FEM-T3-CNG are much lower than the standard FEM-T3 for all propagation directions. The reason for this might be that the acoustic pressure gradient fields from the present FEM-T3-CNG are much smoother than those from the standard FEM-T3, and therefore, much more numerical results can be obtained.

Furthermore, the percentage amplitude decays of the acoustic wave are also calculated and compared in Figure 4. It is seen that the results from FEM-T3 are clearly larger than those from the present FEM-T3-CNG. More importantly, we can also find that the numerical anisotropy properties of the solutions can be largely controlled by the present FEMT3-CNG, namely, the accuracy of FEM-T3-CNG solutions seem to be not dependent on the propagation directions, while the FEM-T3 solutions are quite dependent on the propagation directions.

4.2. Acoustic Scattering by the Infinite Length Rigid Cylinder. As shown in Figure 5, the infinite length rigid cylinder with radius $a=0.2$ is fixed in the unbounded problem domain and the surrounding uniform medium has a density $\rho=1000 \mathrm{~kg} / \mathrm{m}^{3}$ and acoustic wave speed $c=1000 \mathrm{~m} / \mathrm{s}$. The incident acoustic plane wave is travelling along the $x$-axis, and the unbounded problem domain is truncated by introducing a circular artificial boundary with radius $R=1.2$.

The scattered acoustic pressure distributions at a distance $l=1 \mathrm{~m}$ from the origin are calculated and compared here to demonstrate the good features of the present FEMT3-CNG element for underwater acoustic scattering 


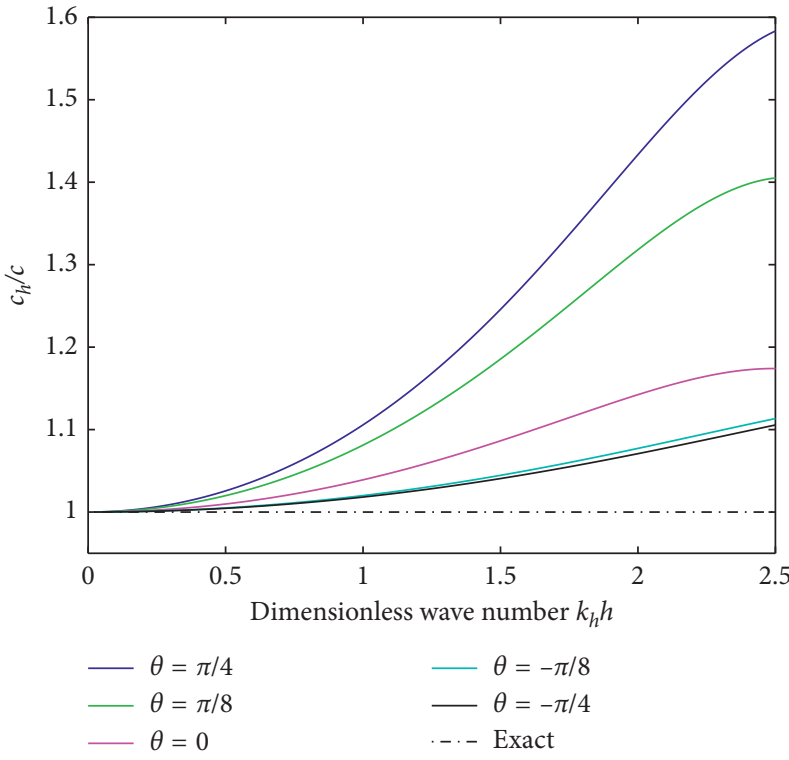

(a)

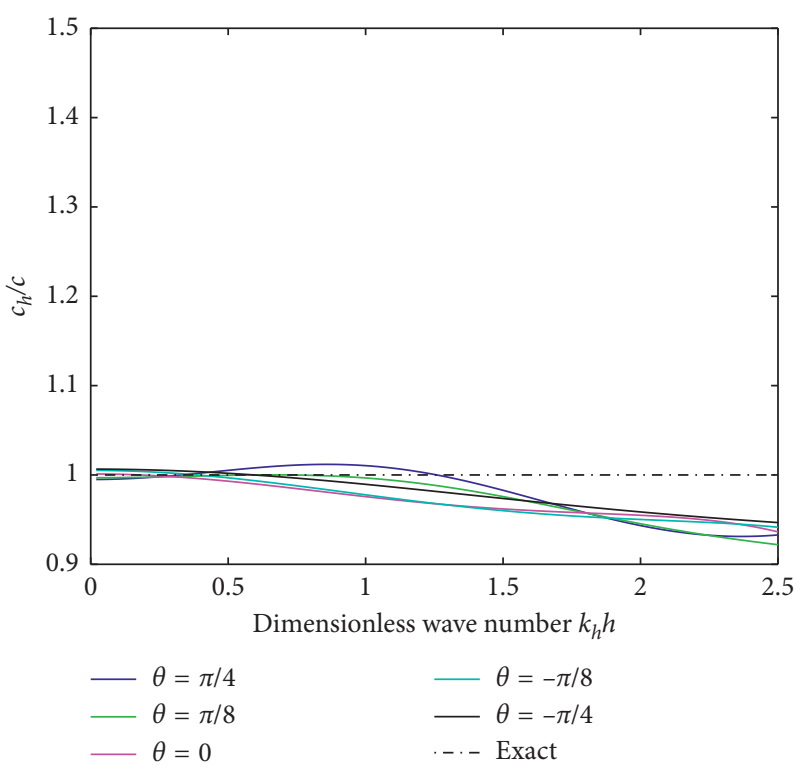

(b)

Figure 3: The dispersion properties of FEM-T3 and FEM-T3-CNG for different propagation directions $(\mathrm{CFL}=1)$ : $(\mathrm{a})$ FEM-T3 and (b) FEM-T3-CNG.

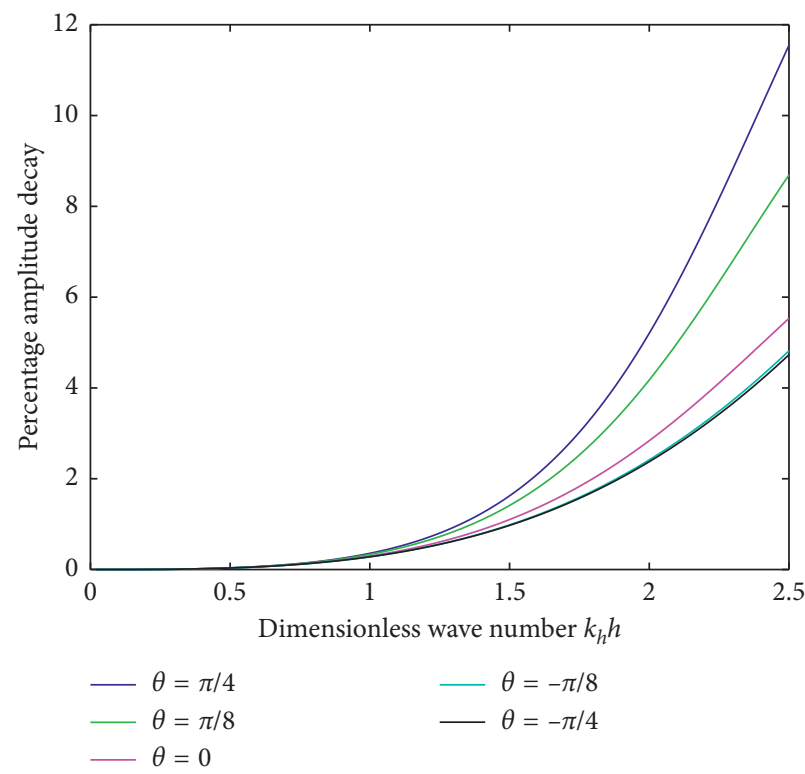

(a)

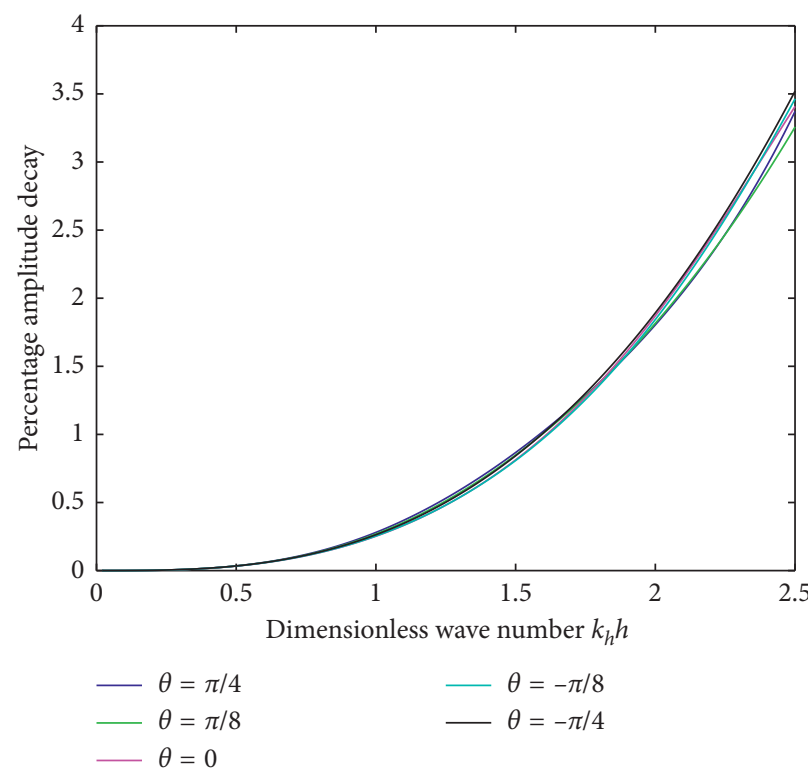

(b)

FIGURE 4: The percentage amplitude decays of the acoustic wave for different propagation directions (CFL=1): (a) FEM-T3 and (b) FEMT3-CNG.

problems. To make the comparison more clear, three different wave numbers $(k=\pi, k=3 \pi$, and $k=6 \pi)$ are employed in the calculation process, and the computational problem domain is divided into 563 nodes and 1030 triangular elements. Figure 6 shows the comparisons of the acoustic scattering pattern using FEM-T3, Galerkin least-squares (GLS) FEM [32], and the present FEM-T3-CNG. It is observed that the present FEM-T3-CNG element is able to provide much more accurate solutions than the standard FEM-T3 and the GLS element for solving acoustic scattering problems, in particular, in the relatively large wave numbers. In Figures 7 and 8, we also show the contours of the scattered pressure distributions at two different wave numbers $(k=2 \pi$ and $k=4 \pi$ ). Similar to the previous analysis, the FEM-T3 and FEM-T3-CNG have the similar computation accuracy for the relatively low wave number $(k=2 \pi)$. However, when 


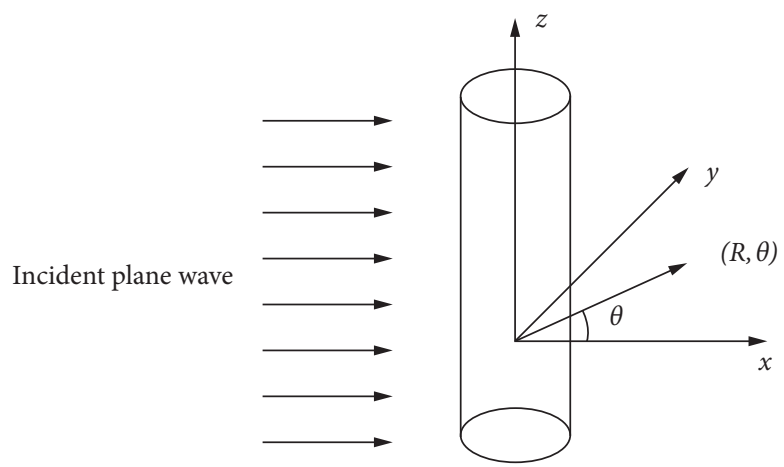

FIgURE 5: Acoustic scattering by an infinite length cylinder.

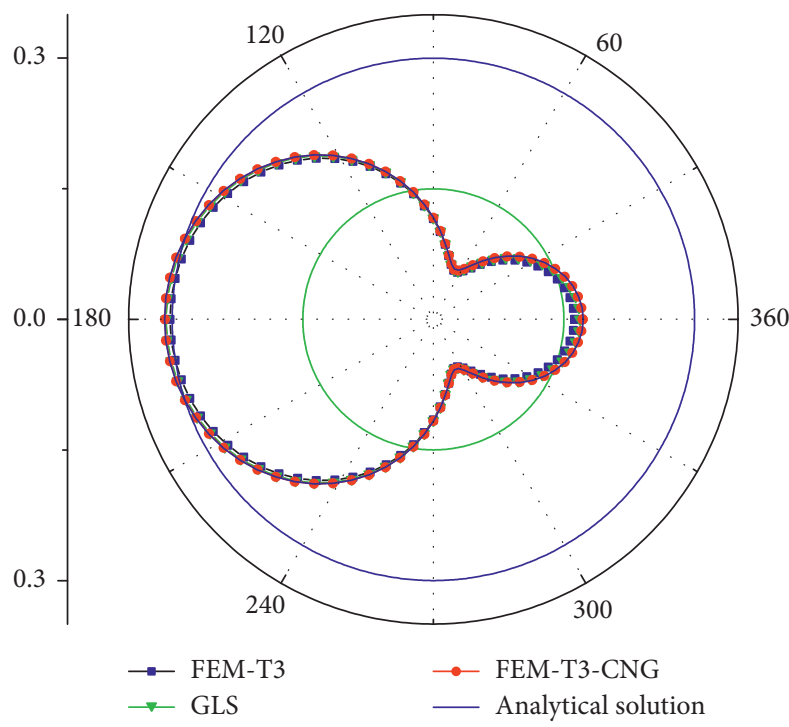

(a)

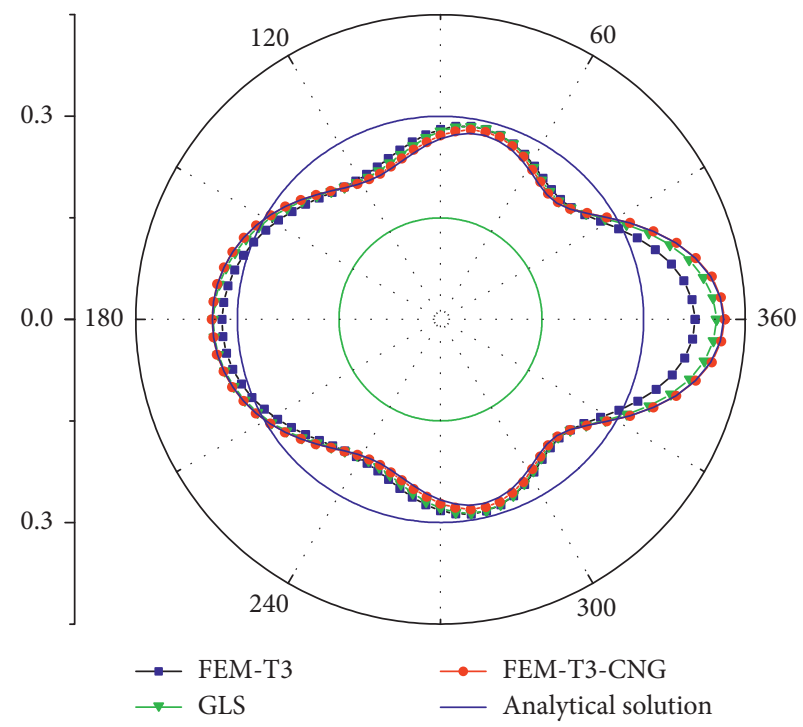

(b)

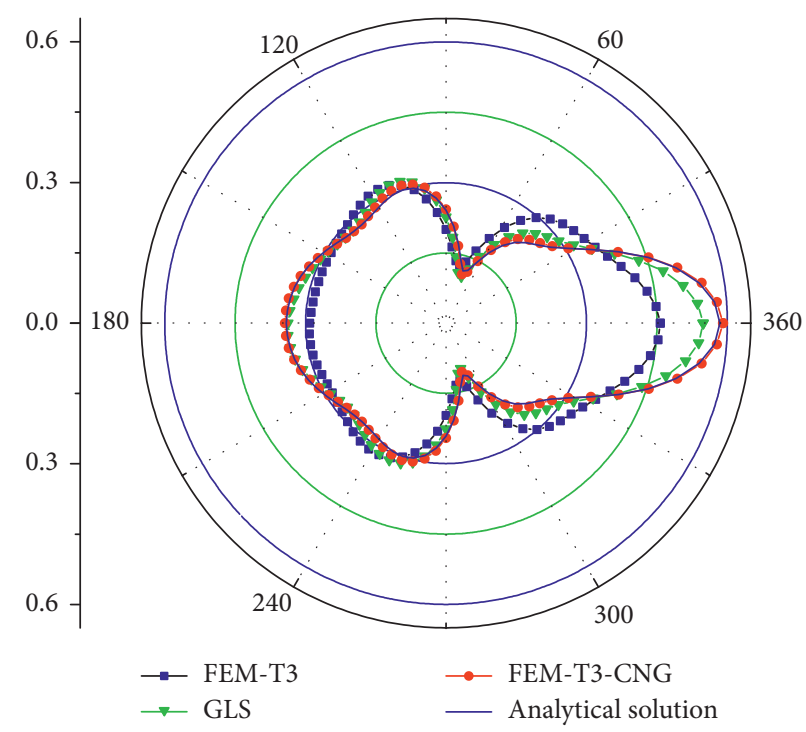

(c)

FIgURE 6: Acoustic scattering pattern from different schemes: (a) $k=\pi$; (b) $k=3 \pi$; (c) $k=6 \pi$.

it comes to the relatively high wave number $(k=4 \pi)$, the accuracy of the present FEM-T3-CNG clearly surpasses the standard FEM-T3 and could achieve better numerical solutions. These findings again demonstrate that the present FEM-T3-CNG is a very promising numerical approach for solving acoustic scattering problems. 


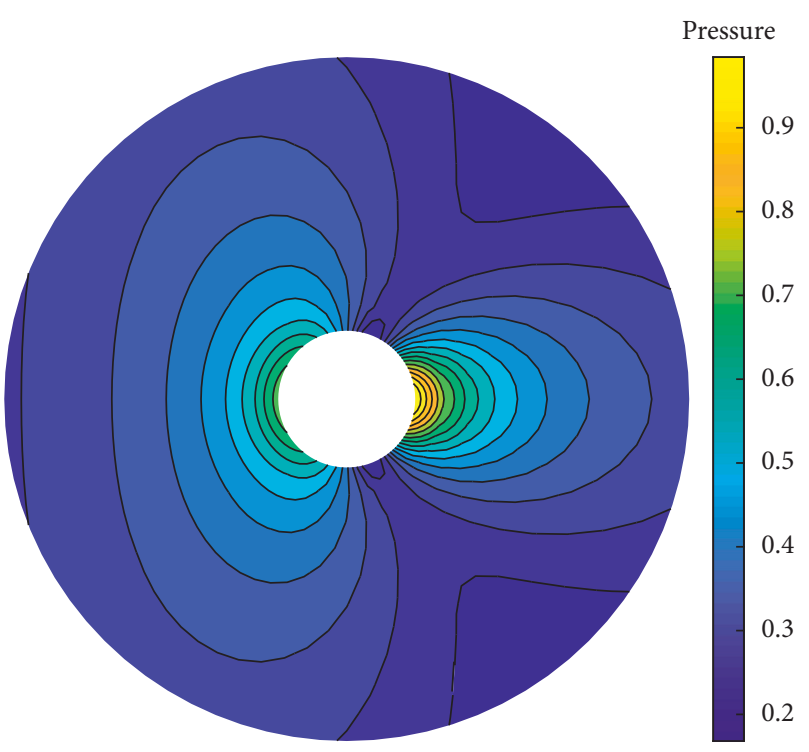

(a)

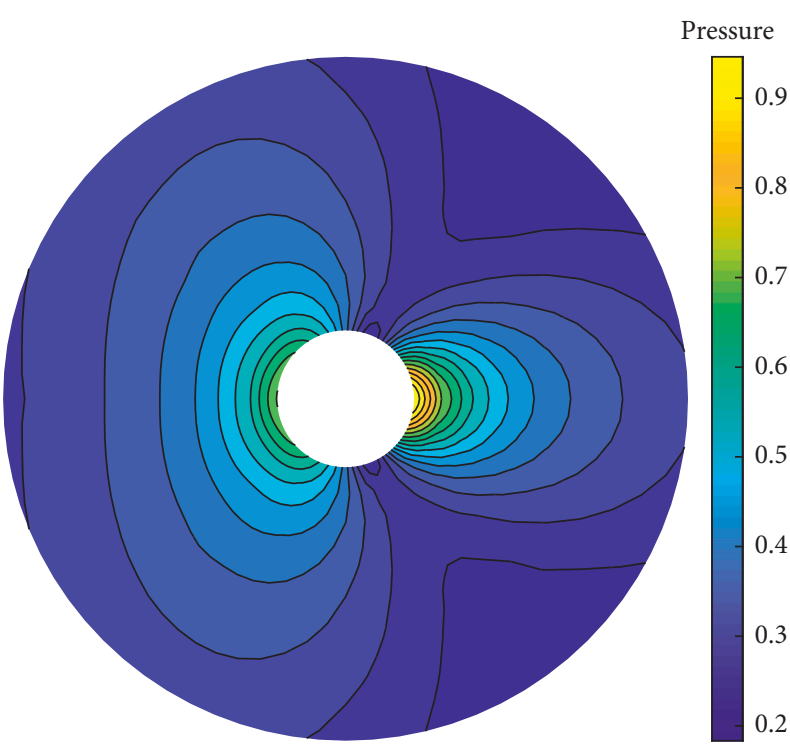

(b)

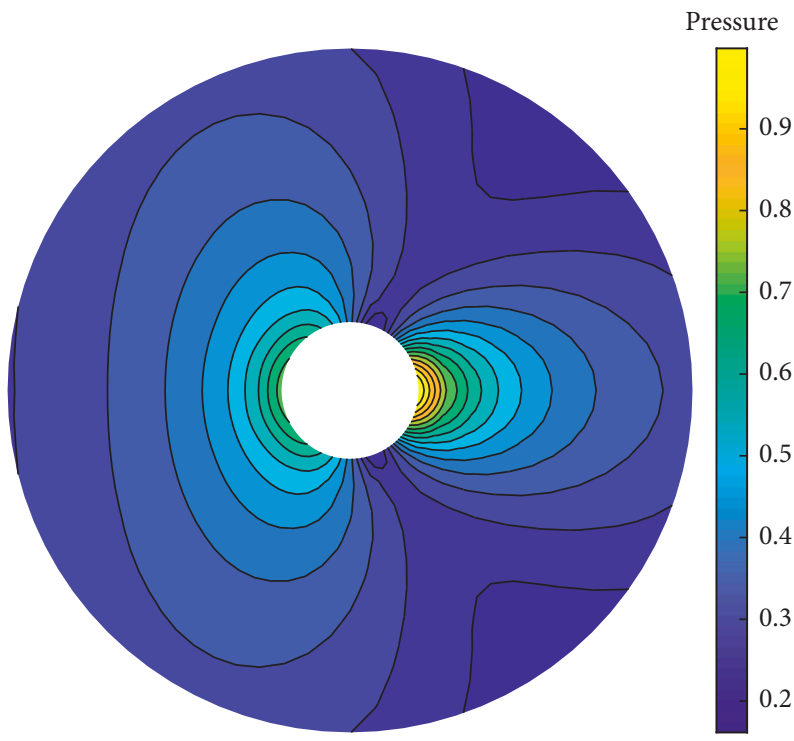

(c)

FIgURE 7: Comparisons of the contour of the scattered pressure distributions at wave number $k=2 \pi$ : (a) exact solution; (b) FEM-T3; (c) FEM-T3-CNG.

From the aforementioned numerical results, we can find that the present-T3-CNG indeed behaves better than the GLS and the standard linear FEM-T3 element in terms of computation accuracy for acoustic scattering. However, whether the FEM-T3-CNG also has the relatively high computation efficiency is still unclear. With this in mind, we here focus on investigating the computation efficiency of the mentioned different elements. To achieve this, a series of meshes with different average mesh sizes are employed in the computation. For a fixed wave number $k=4 \pi$, the total required CPU time for different elements against the numerical error indicator is shown in Figure 9. The used numerical error indicator is defined by

$$
E_{p}=\int_{\Omega}\left(\widetilde{v}^{\mathrm{e}}-\widetilde{v}^{\text {num }}\right)^{T}\left(v^{\mathrm{e}}-v^{\text {num }}\right) \mathrm{d} \Omega,
$$

in which the superscripts " $e$ " and "num" stand for the numerical solutions and the exact solutions, respectively, and the sign " " stands for the complex conjugates of the corresponding variables.

From the numerical results shown in Figure 9, it is seen that the present FEM-T3-CNG is indeed numerically more expensive than the standard linear FEM-T3 and GLS elements. This is because in the FEM-T3-CNG element, more nodes are used to form the element stiffness matrix and element mass matrix. As a result, the bandwidth of the global system matrix from the FEM-T3-CNG is generally larger 


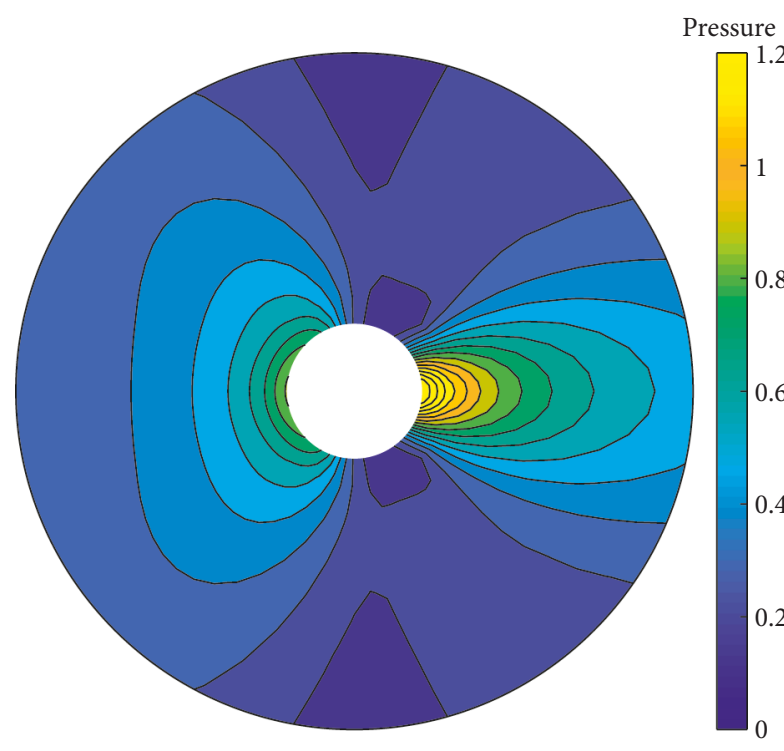

(a)

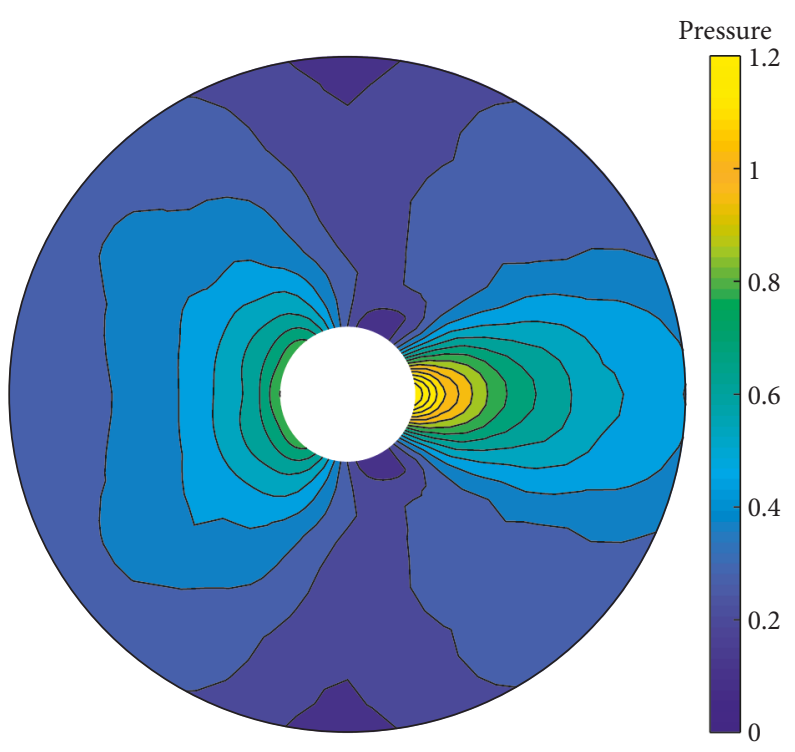

(b)

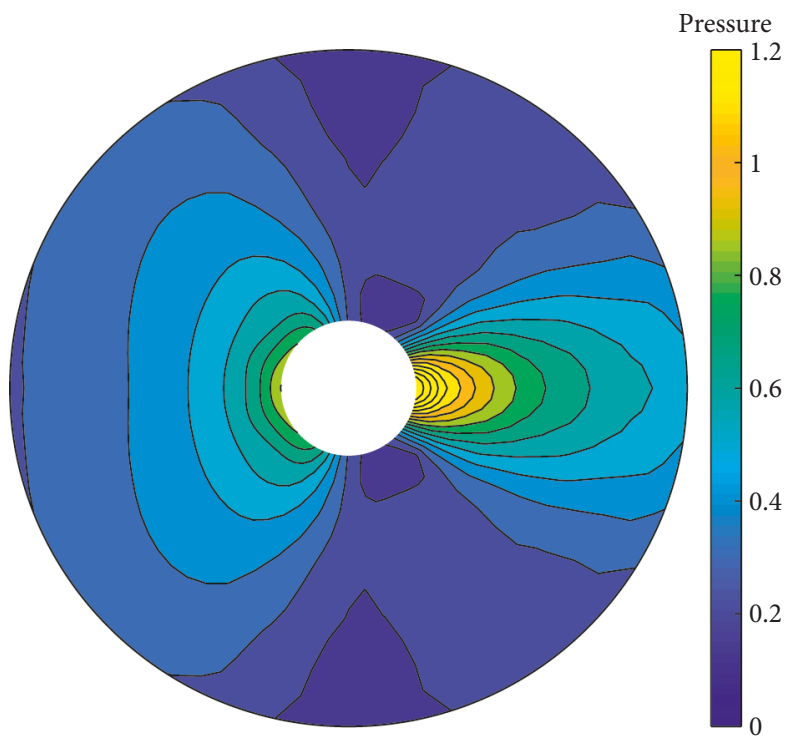

(c)

Figure 8: Comparisons of the contour of the scattered pressure distributions at wave number $k=4 \pi$ : (a) exact solution; (b) FEM-T3; (c) FEM-T3-CNG.

than that from the other two mentioned elements. Besides, since the high order interpolation is employed in the local approximation, the additional Gauss integration points are also responsible for the relatively expensive computational cost. Nevertheless, we can observe that the present FEM-T3CNG still possesses the higher computation efficiency if the computation accuracy is taken into consideration. In other words, to achieve the same computational accuracy, the less computational cost is needed for the present FEM-T3-CNG element compared with the other two elements. Therefore, it is verified that the present FEM-T3-CNG has the relatively high computation efficiency for tackling acoustic scattering problems.
4.3. Acoustic Scattering by an Object with an Arbitrary Geometry Shape. The second numerical example considered here is the acoustic scattering by an object with arbitrary geometry shape. The configuration of this numerical example is shown in Figure 10, and the surrounding acoustic fluid medium is water (density $\rho=1000 \mathrm{~kg} / \mathrm{m}^{3}$ and acoustic wave speed $c=1000 \mathrm{~m} / \mathrm{s})$. The plane acoustic wave is travelling along the positive $x$-axis, and the circular artificial boundary $B$ with radius $R=1$ is centered at the origin of the Cartesian coordinate system. The standard triangular mesh (1174 nodes and 2201 elements) is employed to discretize the considered problem domain and the average mesh size $h$ is $0.06 \mathrm{~m}$. The real part of the scattered acoustic pressure at 


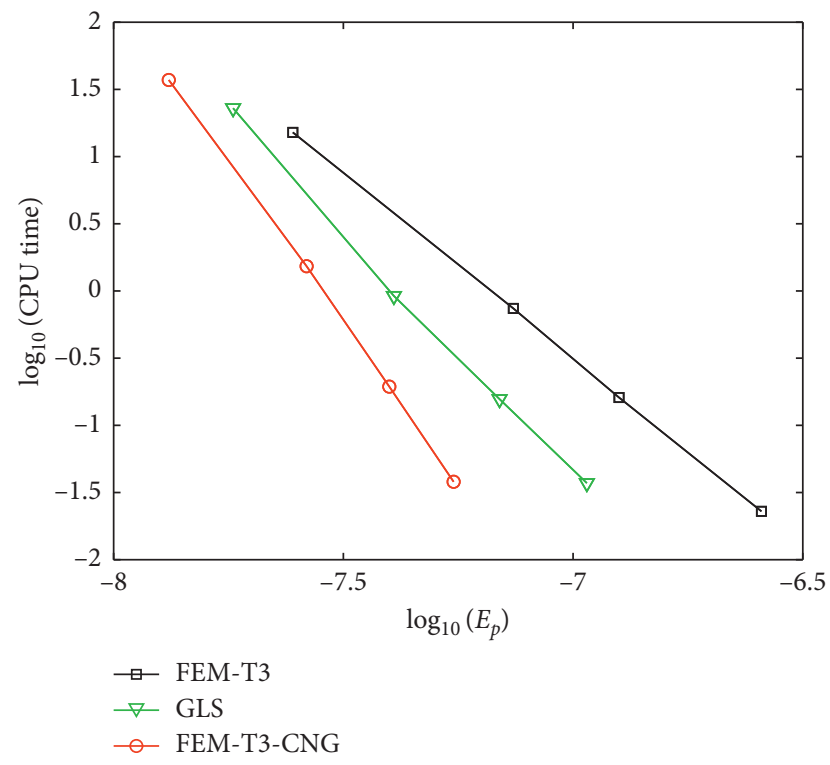

FIgURE 9: Computational cost versus the numerical error indicator for different elements.

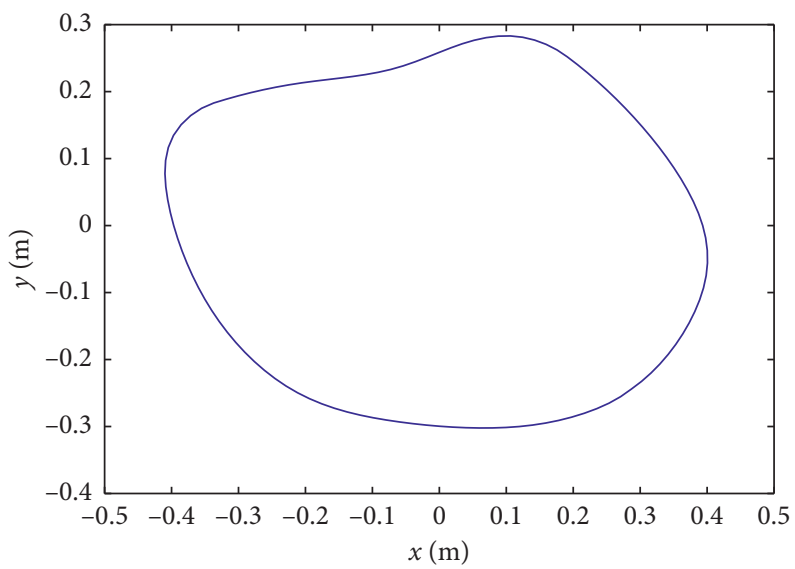

Figure 10: Schematic description of an object with an arbitrary geometry shape.

three different wave number values $(k=10, k=20$, and $k=30$ ) along the $x$-axis are calculated and compared here. The corresponding numerical results from different elements are shown in Figure 11. Due to the unavailableness of the analytical solutions to this acoustic scattering problem, the numerical solutions from the FEM-Q4 element with a very refined mesh (32860 nodes and 64864 elements with average mesh size $h=0.001 \mathrm{~m}$ ) are also given in the figures for comparison. From the figures, it clearly seen that all the three mentioned elements (FEM-T3, GLS, and FEM-T3-CNG) are able to provide similar results which are very close to the reference solutions for the relatively small wave number values $(k=10$ and $k=20)$. However, with the increase of the considered wave number values, the performance of the present FEM-T3-CNG will stand out clearly and can provide much more accurate results than the standard FEM-T3 and GLS. Therefore, it is again demonstrated that the present FEM-T3-CNG element indeed behaves better than the standard FEM-T3 and GLS elements in terms of computation accuracy for exterior acoustic computation.

\section{Conclusions}

This paper focuses on presenting a novel triangular element with continuous nodal acoustic pressure gradient (FEM-T3CNG) for two-dimensional acoustic scattering problems. The dispersion error properties of the present method are firstly investigated and two typical numerical examples are conducted to examine the performance of the present FEMT3-CNG element. The important point for this novel triangular element is that the hybrid nodal shape functions which are constructed by combining the standard finite element (FE) scheme and the least-squares (LS) scheme are used to formulate the discretized form of the governing equation. In addition, since the derivatives of the used shape functions are continuous at nodes, the gradient of the 


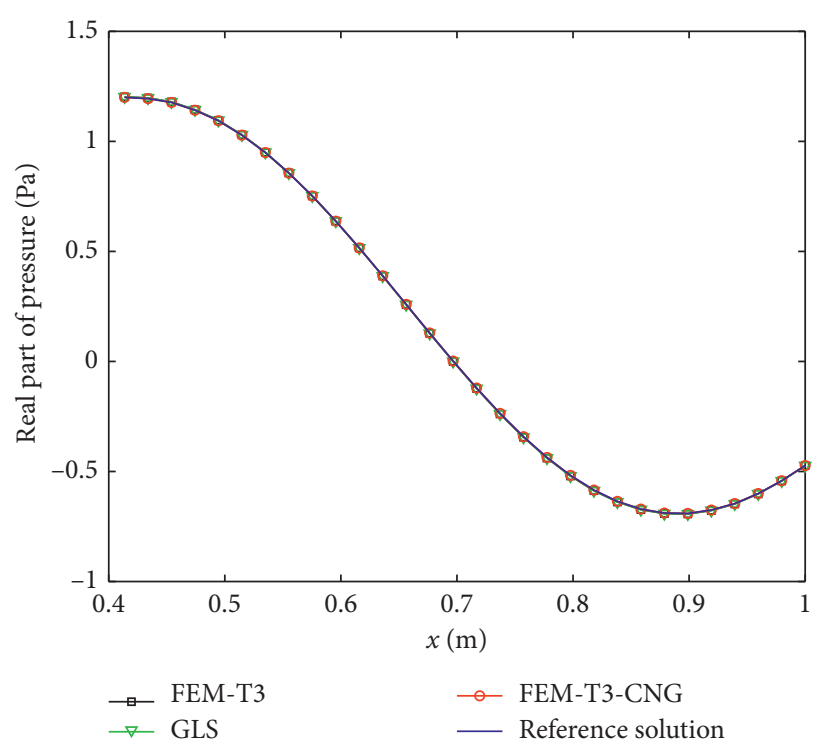

(a)

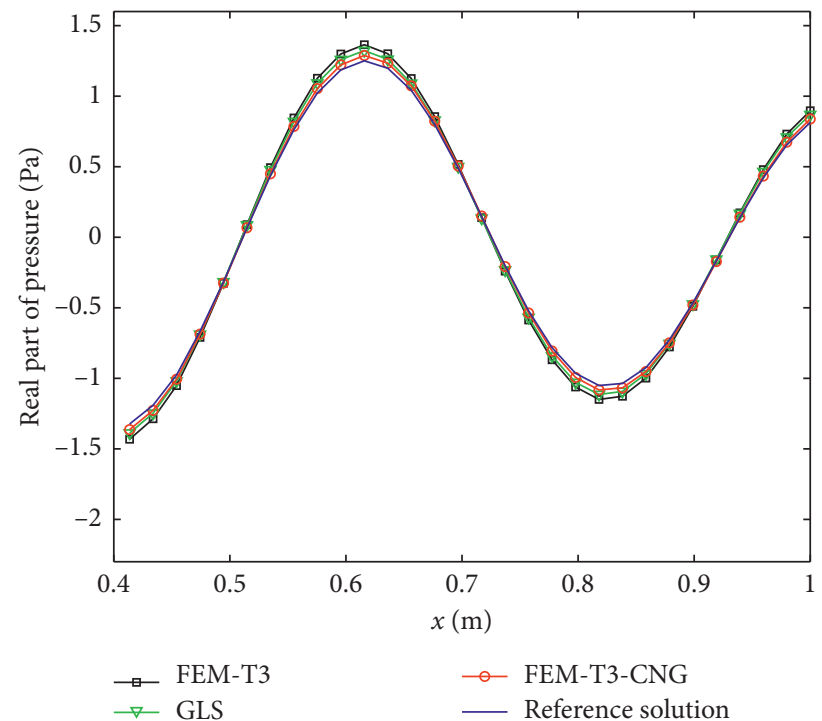

(b)

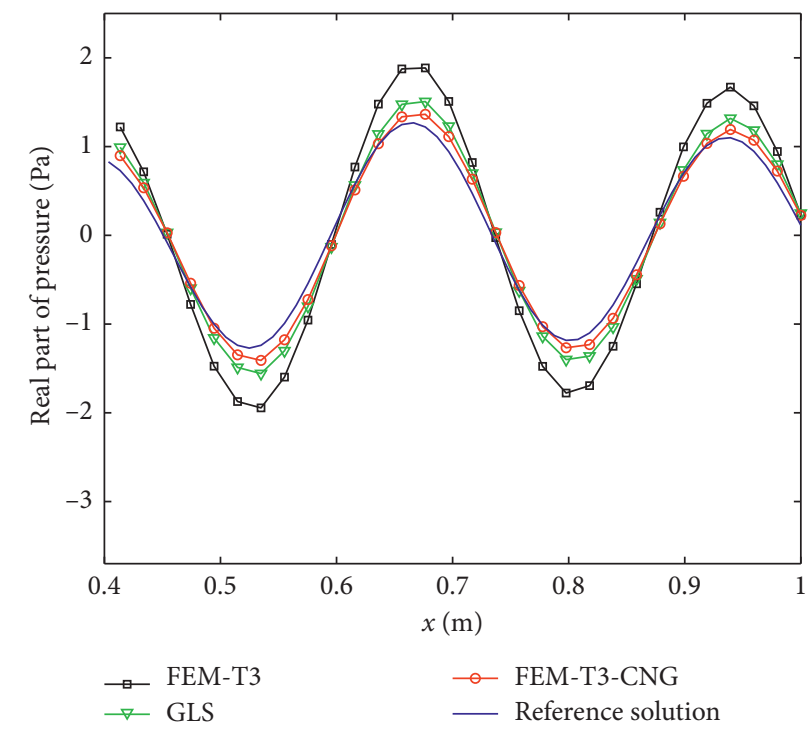

(c)

Figure 11: Comparison of the numerical results from different elements for the object with arbitrary shape: (a) $k=10$; (b) $k=20$; (c) $k=30$.

obtained acoustic pressure field is also continuous at nodes. The numerical results show that the computation accuracy of the standard FEM-T3 element can be significantly improved by the present FEM-T3-CNG element, and it can be a very promising numerical approach for solving practical underwater acoustic scattering problems.

\section{Data Availability}

The data used to support the findings of this study areavailable from the corresponding author upon request.

\section{Conflicts of Interest}

The authors declare that they have no conflicts of interest.

\section{Acknowledgments}

The authors wish to express their gratitude to the National Natural Science Foundation of China (grant number 51579112), Fundamental Research Funds for the Central Universities (grant number WUT: 2019IVB012), and the China Postdoctoral Science Foundation (grant numbers 2018M632866 and 2018M642940).

\section{References}

[1] I. Harari, "A survey of finite element methods for time-harmonic acoustics," Computer Methods in Applied Mechanics and Engineering, vol. 195, no. 13-16, pp. 1594-1607, 2006.

[2] J. Li, W. Chen, and Q. Qin, "A modified dual-level fast multipole boundary element method based on the BurtonMiller formulation for large-scale three-dimensional sound 
field analysis," Computer Methods in Applied Mechanics and Engineering, vol. 340, pp. 121-146, 2018.

[3] Y. Gu, X. He, W. Chen, and C. Zhang, "Analysis of threedimensional anisotropic heat conduction problems on thin domains using an advanced boundary element method," Computers \& Mathematics with Applications, vol. 75, no. 1, pp. 33-44, 2018.

[4] J. Li, Z. Fu, and W. Chen, "Numerical investigation on the obliquely incident water wave passing through the submerged breakwater by singular boundary method," Computers \& Mathematics with Applications, vol. 71, no. 1, pp. 381-390, 2016.

[5] Z. Tang, Z. J. Fu, D. J. Zheng, and J. D. Huang, "Singular boundary method to simulate scattering of $\mathrm{SH}$ wave by the canyon topography," Advances in Applied Mathematics and Mechanics, vol. 10, no. 4, pp. 912-924, 2018.

[6] Z. Fu, W. Chen, P. Wen, and C. Zhang, "Singular boundary method for wave propagation analysis in periodic structures," Journal of Sound and Vibration, vol. 425, pp. 170-188, 2018.

[7] J. Li, W. Chen, Z. Fu, and Q.-H. Qin, "A regularized approach evaluating the near-boundary and boundary solutions for three-dimensional Helmholtz equation with wideband wavenumbers," Applied Mathematics Letters, vol. 91, pp. 5560, 2019.

[8] Y. Sun, "Indirect boundary integral equation method for the cauchy problem of the Laplace equation," Journal of Scientific Computing, vol. 71, no. 2, pp. 469-498, 2017.

[9] J. Li and W. Chen, "A modified singular boundary method for three-dimensional high frequency acoustic wave problems," Applied Mathematical Modelling, vol. 54, pp. 189-201, 2018.

[10] J. Li, W. Chen, Q.-H. Qin, and Z. Fu, "A modified multilevel algorithm for large-scale scientific and engineering computing," Computers \& Mathematics with Applications, vol. 77, no. 8, pp. 2061-2076, 2019.

[11] L. Chen and X. Li, "Boundary element-free methods for exterior acoustic problems with arbitrary and high wavenumbers," Applied Mathematical Modelling, vol. 72, pp. 85103, 2019.

[12] L. Chen, X. Liu, and X. Li, "The boundary element-free method for 2D interior and exterior Helmholtz problems," Computers \& Mathematics with Applications, vol. 77, no. 3, pp. 846-864, 2019.

[13] F. J. Wang, C. M. Fan, Q. S. Hua, and Y. Gu, "Localized MFS for the inverse Cauchy problems of two-dimensional Laplace and biharmonic equations," Applied Mathematics and Computation, vol. 364, Article ID 124658, 2020.

[14] X. Y. You, W. Li, and Y. B. Chai, "A truly meshfree method for solving acoustic problems using local weak form and radial basis functions," Applied Mathematics and Computation, vol. 365, Article ID 124694, 2020.

[15] J. Lin, C. Z. Zhang, L. L. Sun, and J. Lu, "Simulation of seismic wave scattering by embedded cavities in an elastic half-plane using the novel singular boundary method," Advances in Applied Mathematics and Mechanics, vol. 10, no. 2, pp. 322342, 2018.

[16] X. Wei and L. Sun, "Singular boundary method for 3D timeharmonic electromagnetic scattering problems," Applied Mathematical Modelling, vol. 76, pp. 617-631, 2019.

[17] I. Harari and T. J. R. Hughes, "A cost comparison of boundary element and finite element methods for problems of timeharmonic acoustics," Computer Methods in Applied Mechanics and Engineering, vol. 97, no. 1, pp. 77-102, 1992.

[18] J. B. Keller and D. Givoli, "Exact non-reflecting boundary conditions," Journal of Computational Physics, vol. 82, no. 1, pp. 172-192, 1989.
[19] Y. B. Chai, Z. X. Gong, W. Li et al., “Application of smoothed finite element method to two-dimensional exterior problems of acoustic radiation," International Journal of Computational Methods, vol. 15, no. 5, Article ID 1850029, 2018.

[20] Y. Chai, X. You, W. Li, Y. Huang, Z. Yue, and M. Wang, "Application of the edge-based gradient smoothing technique to acoustic radiation and acoustic scattering from rigid and elastic structures in two dimensions," Computers \& Structures, vol. 203, pp. 43-58, 2018.

[21] X. Hu, X. Cui, Q. Zhang, G. Wang, and G. Li, “The stable node-based smoothed finite element method for analyzing acoustic radiation problems," Engineering Analysis with Boundary Elements, vol. 80, pp. 142-151, 2017.

[22] A. Deraemaeker, I. Babuška, and P. Bouillard, "Dispersion and pollution of the FEM solution for the Helmholtz equation in one, two and three dimensions," International Journal for Numerical Methods in Engineering, vol. 46, no. 4, pp. 471-499, 1999.

[23] X. Y. Cui, X. Hu, G. Wang, and G. Y. Li, "An accurate and efficient scheme for acoustic-structure interaction problems based on unstructured mesh," Computer Methods in Applied Mechanics and Engineering, vol. 317, pp. 1122-1145, 2017.

[24] X. Hu, X. Y. Cui, Q. Y. Zhang, G. Wang, and G. Y. Li, "Dispersion error analysis of stable node-based finite element method for the Helmholtz equation," Communications in Computational Physics, vol. 23, no. 3, pp. 795-821, 2018.

[25] Y. Chai, W. Li, Z. Gong, and T. Li, "Hybrid smoothed finite element method for two-dimensional underwater acoustic scattering problems," Ocean Engineering, vol. 116, pp. 129-141, 2016.

[26] X. You, W. Li, and Y. Chai, "Dispersion analysis for acoustic problems using the point interpolation method," Engineering Analysis with Boundary Elements, vol. 94, pp. 79-93, 2018.

[27] Y. B. Chai, X. Y. You, and W. Li, "Dispersion reduction for the wave propagation problems using a coupled "FE-Meshfree" triangular element," Journal of Computational Methods, 2019.

[28] X. Yue, F. Wang, Q. Hua, and X.-Y. Qiu, "A novel space-time meshless method for nonhomogeneous convection-diffusion equations with variable coefficients," Applied Mathematics Letters, vol. 92, pp. 144-150, 2019.

[29] T. Zhang and X. L. Li, "Variational multiscale interpolating element-free Galerkin method for the nonlinear DarcyForchheimer model," Computers \& Mathematics with Applications, 2019, In press.

[30] W. Qu, "A high accuracy method for long-time evolution of acoustic wave equation," Applied Mathematics Letters, vol. 98, pp. 135-141, 2019.

[31] J. Lin, Y. Xu, and Y. H. Zhang, "Simulation of linear and nonlinear advection-diffusion-reaction problems by a novel localized scheme," Applied Mathematics Letters, vol. 99, Article ID 106005, 2020.

[32] I. Harari and T. J. R. Hughes, "Galerkin/least-squares finite element methods for the reduced wave equation with nonreflecting boundary conditions in unbounded domains," Computer Methods in Applied Mechanics and Engineering, vol. 98, no. 3, pp. 411-454, 1992.

[33] Y. Chai, Z. Gong, W. Li, T. Li, and Q. Zhang, "A smoothed finite element method for exterior Helmholtz equation in two dimensions," Engineering Analysis with Boundary Elements, vol. 84, pp. 237-252, 2017.

[34] G. Wang, X. Y. Cui, H. Feng, and G. Y. Li, “A stable nodebased smoothed finite element method for acoustic problems," Computer Methods in Applied Mechanics and Engineering, vol. 297, pp. 348-370, 2015.

[35] W. Li, Z. X. Gong, Y. B. Chai et al., "Hybrid gradient smoothing technique with discrete shear gap method for shell 
structures," Computers \& Mathematics with Applications, vol. 74, no. 8, pp. 1826-1855, 2017.

[36] W. Li, Y. Chai, M. Lei, and T. Li, "Numerical investigation of the edge-based gradient smoothing technique for exterior Helmholtz equation in two dimensions," Computers \& Structures, vol. 182, pp. 149-164, 2017.

[37] W. Li, Y. Chai, M. Lei, and G. R. Liu, "Analysis of coupled structural-acoustic problems based on the smoothed finite element method (S-FEM)," Engineering Analysis with Boundary Elements, vol. 42, pp. 84-91, 2014.

[38] Z. C. He, E. Li, G. R. Liu, G. Y. Li, and A. G. Cheng, "A massredistributed finite element method (MR-FEM) for acoustic problems using triangular mesh," Journal of Computational Physics, vol. 323, pp. 149-170, 2016.

[39] K.-T. Kim, L. Zhang, and K.-J. Bathe, "Transient implicit wave propagation dynamics with overlapping finite elements," Computers \& Structures, vol. 199, pp. 18-33, 2018.

[40] Y. Yang, X. Tang, and H. Zheng, "A three-node triangular element with continuous nodal stress," Computers \& Structures, vol. 141, pp. 46-58, 2014. 


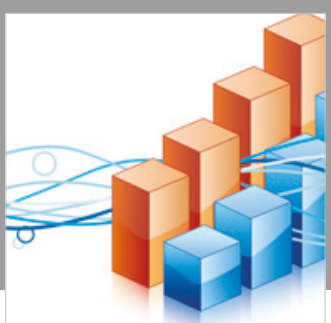

Advances in

Operations Research

\section{-n-m}
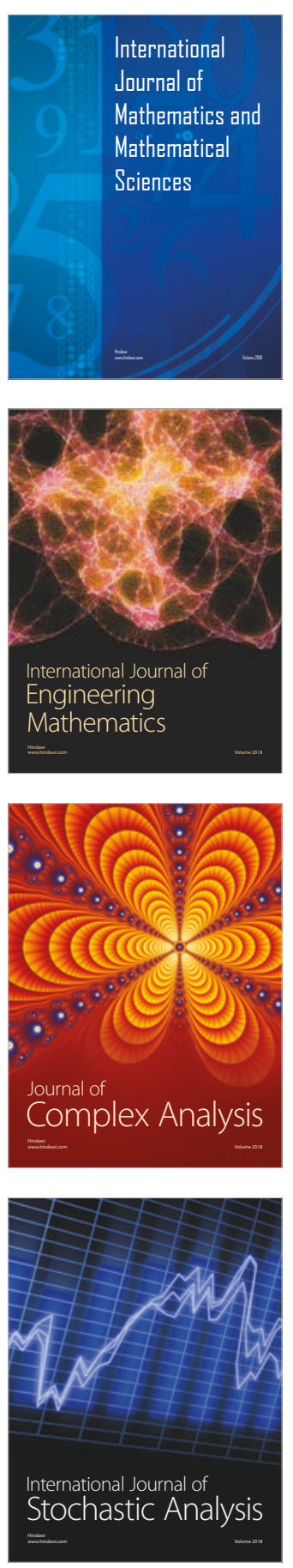
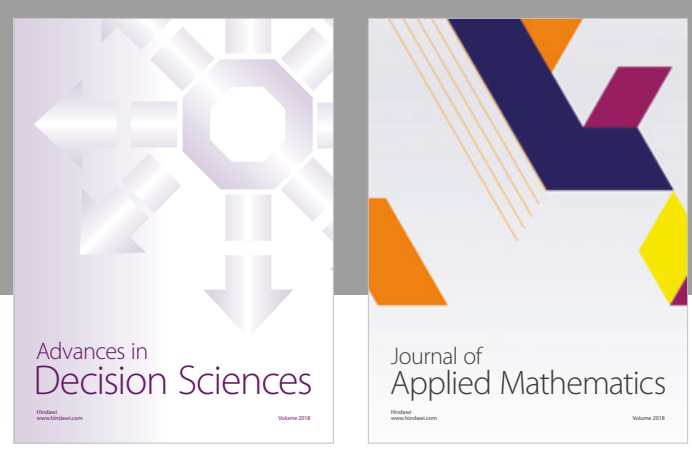

Journal of

Applied Mathematics
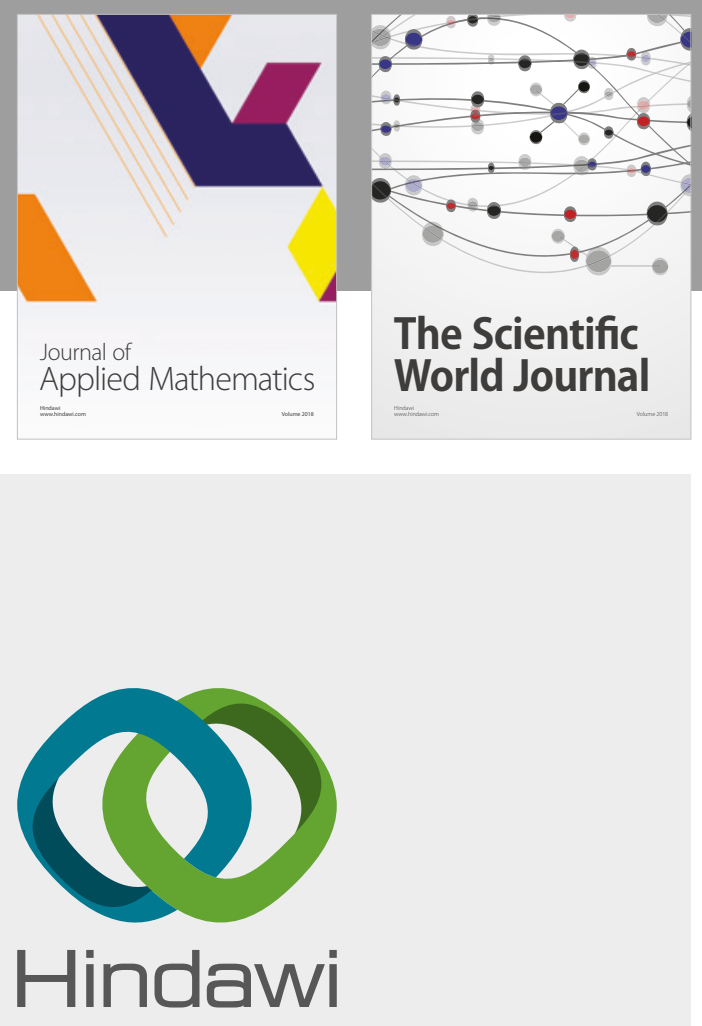

Submit your manuscripts at

www.hindawi.com

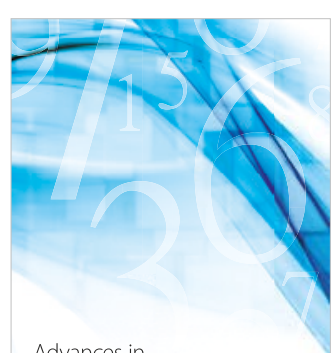

Advances in
Numerical Analysis
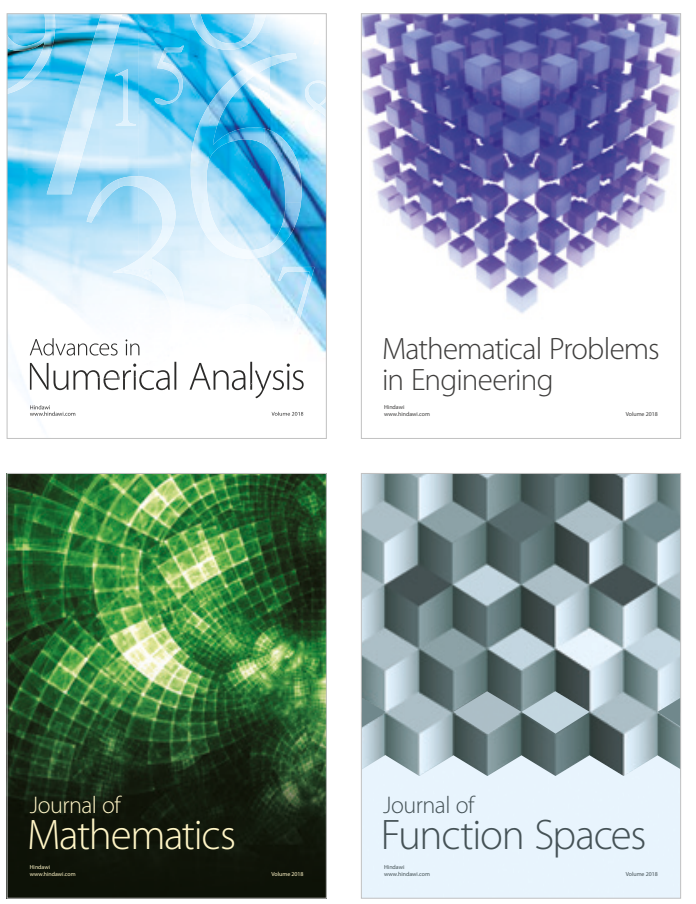

Mathematical Problems in Engineering

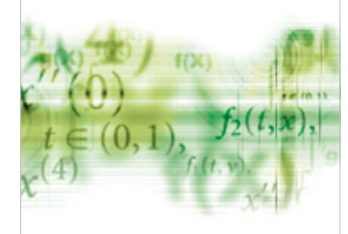

International Journal of

Differential Equations

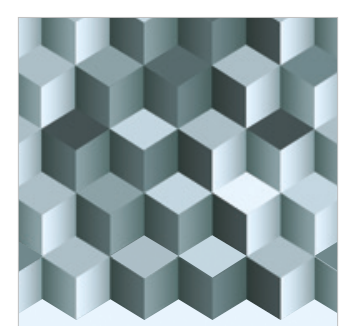

Journal of

Function Spaces

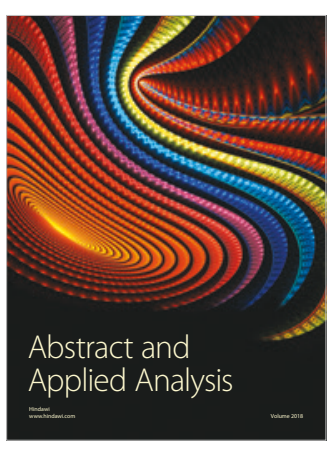

The Scientific

World Journal

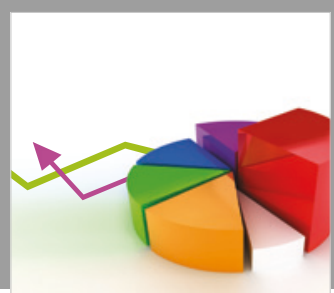

Journal of

Probability and Statistics
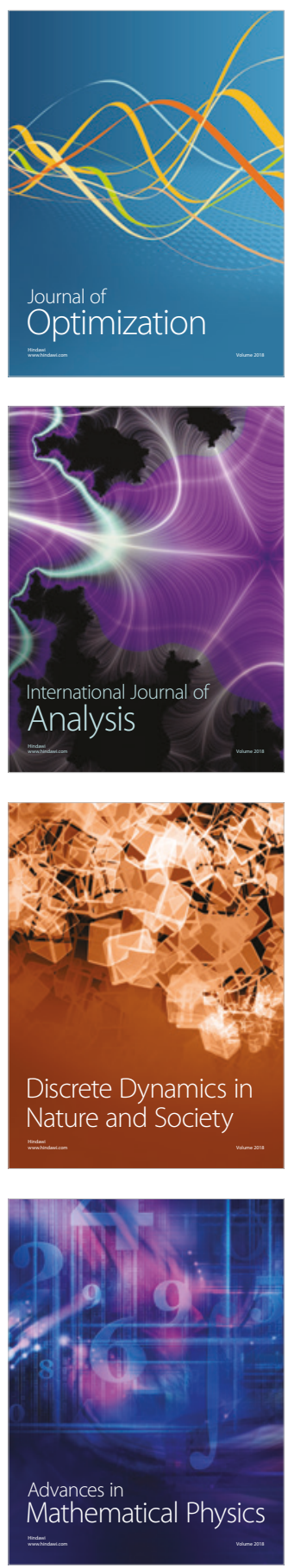